\title{
Correction to: Memory Modalities Opening-up Digital Heritage Infrastructures
}

\author{
Gertraud Koch (iD
}

\section{Correction to:}

Chapter "Memory Modalities Opening-up Digital Heritage Infrastructures" in: M. Rauterberg (Ed.):

Culture and Computing, LNCS 12795, https://doi.org/10.1007/978-3-030-77431-8_15

In the originally published version of chapter 15 , reference 33 contained an error in the DOI. This has now been corrected. 